\title{
VHE BL Lacs through the MAGIC glasses
}

\author{
Josefa Becerra González ${ }^{1,2,3}$ \\ on behalf of the MAGIC Collaboration \\ ${ }^{1}$ NASA Goddard Space Flight Center, Greenbelt, MD 20771, USA \\ ${ }^{2}$ Inst. de Astrofísica de Canarias, E-38200 La Laguna, Tenerife, Spain \\ ${ }^{3}$ Department of Physics and Department of Astronomy, University of Maryland, \\ College Park, MD 20742, USA \\ email: josefa.becerra@nasa.gov
}

\begin{abstract}
In this contribution an overview of the latest results on the study of BL Lac objects with the MAGIC telescopes at the very high energy (VHE, E $>100 \mathrm{GeV}$ ) gamma-rays is presented. Three new VHE sources were detected during 2014, two BL Lac objects and the gravitational lensed blazar S3 0218+357. MAGIC detected very fast intra-night variability from IC 310. This detection points to smaller emitting regions than the event horizon, this is hard to be explained in the framework of the current theoretical models. The long term multi wavelength (MWL) study of the BL Lac PKS 1424+240 shows correlation between the radio and optical emission, pointing to a common origin. The MWL SED is not well fitted by a one-zone synchrotron-self Compton (SSC) model, but a two-zone SSC model can explain both, the MWL light curve and the SED. Spectral curvature has been found in the observed VHE spectrum from PG 1553+113. This is the first time that spectral curvature compatible with the EBL absorption is found in an individual object.
\end{abstract}

Keywords. gamma rays: observations, BL Lacertae objects: general

\section{The MAGIC telescopes}

The MAGIC system consists of two $17 \mathrm{~m}$-diameter Imaging Atmospheric Cherenkov Telescopes (IACT) located on the Roque de los Muchachos, Canary Island of La Palma $\left(28^{\circ} 46^{\prime} \mathrm{N}, 17^{\circ} 53^{\prime} \mathrm{W}\right)$, at a height of $2200 \mathrm{~m}$ above see level. MAGIC observes cosmic gamma rays in the energy range between $50 \mathrm{GeV}$ and $>10 \mathrm{TeV}$ (known as Very High Energy or VHE band). The system reaches a sensitivity of $(0.66 \pm 0.03) \%$ of the Crab Nebula flux for $E>220 \mathrm{GeV}$ in $50 \mathrm{~h}$ of observations (Aleksić et al. 2014a). The energy threshold (defined as the peak of the energy distribution of triggering gamma rays) of MAGIC is $50 \mathrm{GeV}$. The system reaches an angular resolution of $0.07^{\circ}$ at $300 \mathrm{GeV}$. The best spectral resolution of $16 \%$ is reached at a few hundred $\mathrm{GeV}$.

\section{New members of the VHE family}

The VHE family is still very limited, and only $\sim 60$ extragalactic emitters have been detected so far. The sample is highly dominated by BL Lacs objects, while there are only four flat spectrum radio quasars (FSRQs). During 2014, three new VHE emitters have been detected with the MAGIC telescopes: the BL Lac objects RBS 0723 (Mirzoyan et al. 2014a) and RX J1136.5+6737 (Mirzoyan et al. 2014b) and the FSRQ S3 0218+357 (Mirzoyan et al. 2014c).

RBS 0723 is a BL Lac object at redshift $\mathrm{z}=0.198$. MAGIC started the observations of the source on 2013 December 3. The data taken between 2014 Jan 1 and Jan 8 (about 10 hours) yields a source detection with a statistical significance of more than 5 standard 
deviations (Mirzoyan et al. 2014a). The non-detection in the earlier data taken in 2013 Dec. suggests VHE flux variability. The VHE emission measured with MAGIC has a flux of about $2.5 \%$ of the Crab nebula at energies above $200 \mathrm{GeV}$.

RX J1136.5+6737 is a BL Lac object located at $\mathrm{z}=0.1342$. The source was observed with the MAGIC telescopes starting on 2014 January 29. Data taken up to 2014 April 3 (about 20 hours in total) yielded a source detection with a statistical significance of more than 5 standard deviations (Mirzoyan et al. 2014b). The averaged flux measured by MAGIC during the 2014 observations corresponded to about $1.5 \%$ of the Crab nebula at energies above $200 \mathrm{GeV}$. RX J1136.5+6737 is an X-ray bright high-peaked frequency BL Lac object as listed in the MAXI 3-year catalog (Hiroi et al. 2013). RX J1136.5+6737 has also been detected by Fermi-LAT, in the 2FGL catalog (Nolan et al. 2012) with a hard photon index $1.68 \pm 0.12$, and belongs to the first Fermi-LAT catalog of $>10 \mathrm{GeV}$ sources (1FHL; Ackermann et al. 2013), showing bright emission above $10 \mathrm{GeV}$.

$\mathrm{S} 30218+357$ is a gravitationally lensed blazar located at $\mathrm{z}=0.94$. It is the only system where the gravitational lens effects have been clearly detected in gamma-rays, first detected by Fermi-LAT at high energy (HE, E=100 MeV-100 GeV) (Cheung et al. 2014). Recently, in July 2014 the source was detected in high state by the Fermi-LAT (Buson et al. 2014). Due to moon constraints the source could not be observed by MAGIC. However, the observations were scheduled 10 days after the initial flare observed by Fermi-LAT, to cover time period in which delayed emission was expected. The source was detected with the MAGIC telescopes during the delayed emission phase between 2014 July 23 to 26 (Mirzoyan et al. 2014c). In addition to the interesting study of a gravitational lensed object by first time in the VHE band, this source set a new redshift record in the VHE range which makes S3 0218+357 an ideal target for extragalactic background light (EBL) studies.

\section{Short term variability of the active nucleus of IC $\mathbf{3 1 0}$}

IC $310(\mathrm{z}=0.01898)$ was first classified as a head-tail radio galaxy (Ryle \& Windram 1968). However, a more recent analysis of the archival VLBA data of IC 310 shows a blazar-like parsec-scale structure (Kadler et al. 2012). The source was detected at high energies above $30 \mathrm{GeV}$ with Fermi-LAT (Neronov et al. 2010) as well as at very high energies above $260 \mathrm{GeV}$ with the MAGIC Telescopes (Aleksić et al. 2010). IC310 was detected by MAGIC for the first time from October 2009 to February 2010 as part of the Perseus cluster observations. IC 310 is located $0.6^{\circ}$ from the cluster center. Since the observations were performed in the so-called wobble mode, i.e. pointing alternatively to two positions $0.4^{\circ}$ away (along the RA axis) from the cluster center (NGC 1275), IC 310 was observed off-axis. The position of the source changes from $0.25^{\circ}$ to $1^{\circ}$ from the camera center in the two wobble positions respectively. The analysis of the closest wobble position detected the source with a flux compatible with a steady emission. However, an improved analysis was carried out using the data from both wobble positions providing more accurate results and resulted in a clear detection of night-to-night variability at confidence level $>5 \sigma$ (Aleksić et al. 2014b).

Later on, an exceptionally bright flare of IC 310 was detected in November 2012 reaching a flux level of up to $>0.5$ Crab units above $300 \mathrm{GeV}$ during one night (see Fig. 1). Fast intra-night variability was detected during the flare (Aleksic et al. 2014c). This is among the fastest variability ever detected in a blazars, together with Mrk 501 (Albert et al. 2007) and PKS 2155-304 (Aharonian et al. 2007). However, in the later two cases the emission is Doppler-shifted by a larger factor than in IC 310 for which the largest 


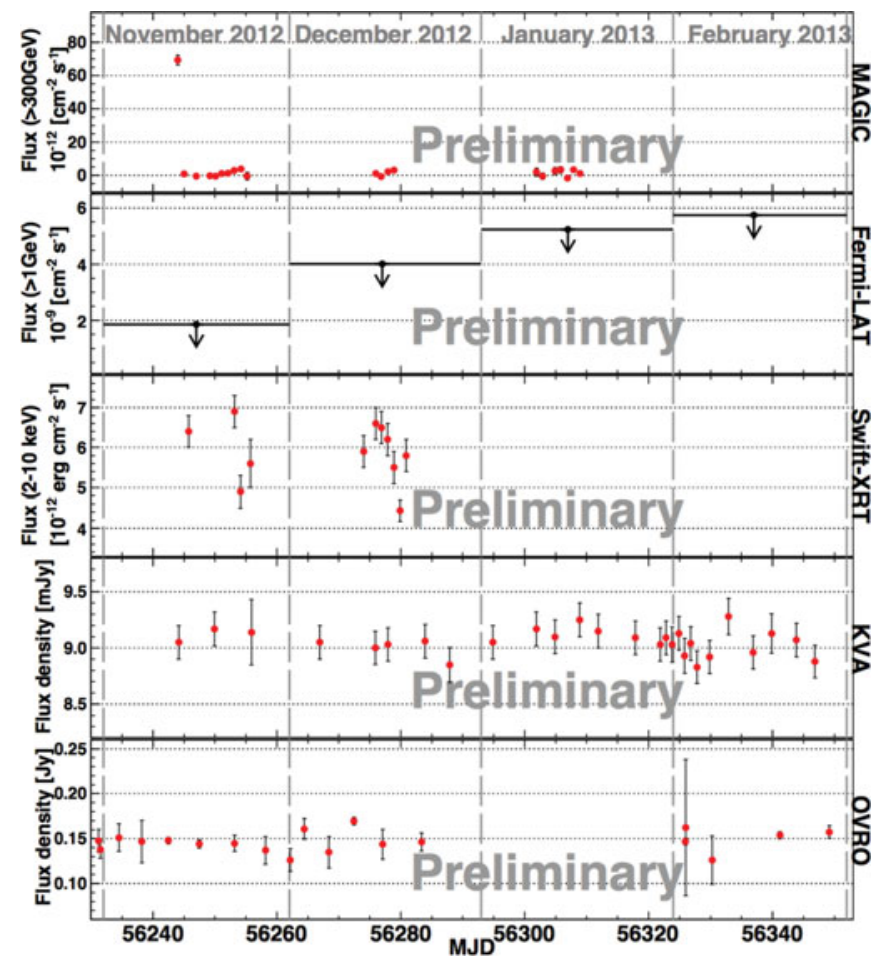

Figure 1. MWL light curve of IC 310: MAGIC above $300 \mathrm{GeV}$, Fermi-LAT above $1 \mathrm{GeV}$, Swift-XRT, KVA R-band data and, OVRO at $15 \mathrm{GHz}$ from November 2012 to February 2013. The optical data from KVA presented here are not host-galaxy corrected.

possible Doppler factor is around 6. The intrinsic variability of the source may in fact be so fast that the emission region is smaller than the event horizon light-crossing time.

\section{Multiwavelength study of the distant blazar PKS $1424+240$}

The MAGIC telescopes observed the BL Lac PKS 1424+240 from 2009 to 2011 (Aleksić et al. 2014d). Although its redshift is unknown, it is one of the most distant VHE blazars with a redshift lower limit of $z \geqslant 0.6$ (Furniss et al. 2013). The differential spectra measured during the different campaigns can be described by steep power laws with the indices ranging from $3.5 \pm 1.2$ to $5.0 \pm 1.7$ (Aleksić et al. 2014d). The MAGIC spectra corrected for the absorption due to the EBL connect smoothly, within systematic errors, with the mean spectrum in 2009-2011 observed at lower energies by the Fermi-LAT. The EBLcorrected MAGIC spectrum is flat with no apparent turn down up to $400 \mathrm{GeV}$. The MWL light curve shows an increase of the flux in radio and optical bands, and both bands shows correlation in the long term. This could point to a common origin from the same region of the jet, unlike the typical assumption in the canonical one-zone emitting zone where the radio emission is assumed to be produced in a different region of the jet.

The MWL SED shows a two peak distribution with a large separation between the two peaks. The one-zone synchrotron self-Compton (SSC) model requires an extremely high Doppler factor which is much higher than the typical values for blazars. Moreover, as shown in Fig. 2 the one-zone model cannot explain properly the observed spectral shape in optical-UV (after subtraction of the host galaxy). However, a two-component synchrotron self-Compton model describes the SED of the source well with reasonable 

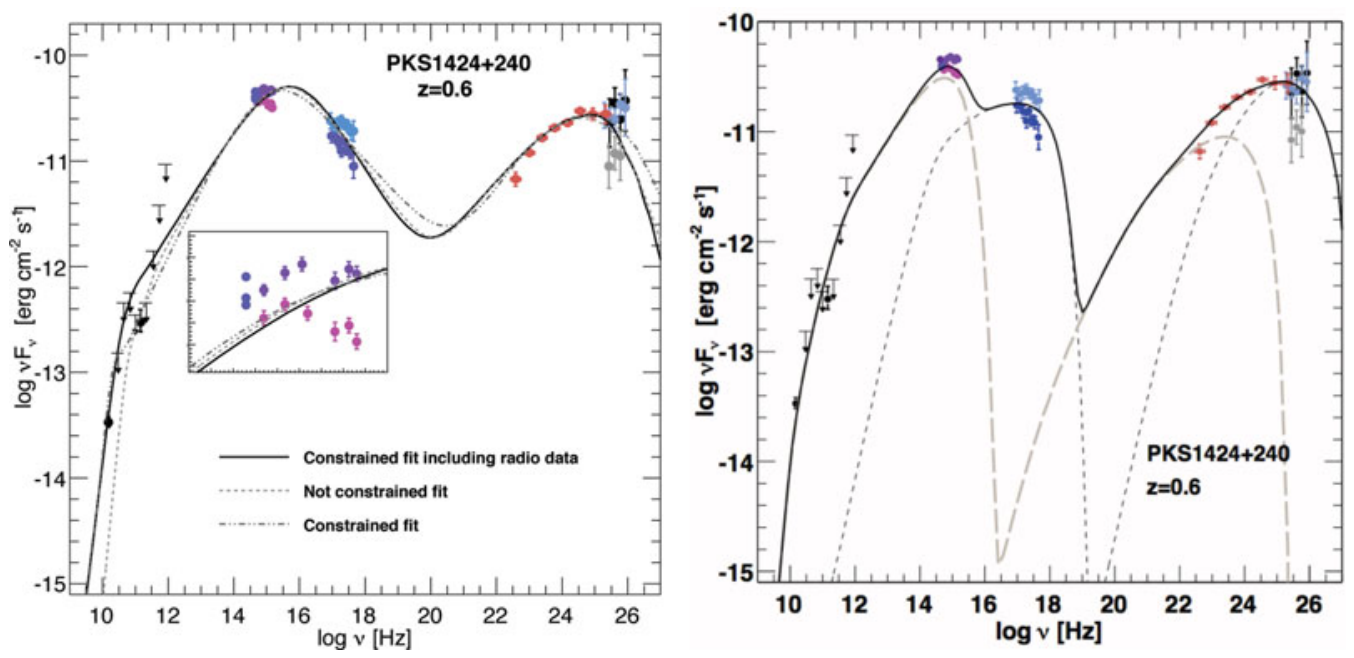

Figure 2. Left panel: SED of PKS $1424+240$ fitted with three single-zone SSC models: the high $\gamma_{\min }$ fit (solid line) and the fits that result from the $\chi^{2}$ minimization (dashed and dot-dashed lines). Right panel: SED of PKS 1424+240 fitted with a two-zone SSC model. The long dashed line represents the emission from the outer region and the dashed line from the inner region. In both cases it has been assumed a redshift of $\mathrm{z}=0.6$. Data from radio to VHE: OVRO (black filled circles), Planck (black arrows and filled circle), optical R-band from KVA (blue filled circles), UVOT (pink (lowest state) and purple (highest state) filled circles), Swift-XRT (light blue (high state) and violet (low state) filled circles), Fermi-LAT (red filled circles) and MAGIC (2009: black, 2010: gray, 2011: light blue).

Doppler factor parameters, and is in agreement with the observed multi wavelength flux evolution behavior.

\section{Probing the VHE spectral curvature}

PG $1553+113$ is a VHE $\gamma$-ray emitter classified as a BL Lac object. Its redshift is constrained by intergalactic absorption lines in the range $0.4<z<0.58$ (Danforth et al. 2010). The MAGIC telescopes have monitored the source's activity since 2005. In early 2012, PG 1553+113 was found in a high-state, and later, in April of the same year, the source reached its highest VHE flux state detected so far (Aleksić et al. 2014e). Simultaneous observations carried out in X-rays during 2012 April show similar flaring behaviour. In contrast, the $\gamma$-ray flux at $E<100 \mathrm{GeV}$ observed by Fermi-LAT is compatible with steady emission.

The VHE spectrum shows clear curvature and a power-law fit can be discarded with confidence level of $4.7 \sigma$. Instead, the observed VHE spectrum can be well fitted by either a power-law with an exponential cut-off or by a log-parabola. For the first time spectral curvature compatible with the EBL imprint (according to the present generation of EBL models) was found in an individual source (Fig. 3).

New constraints on the redshift were derived from the VHE spectrum. These constraints are compatible with previous limits and suggest that the source is most likely located around the optical lower limit, $z=0.4$, based on the detection of Ly $\alpha$ absorption (Danforth et al. 2010). The MWL SED during the flaring episode is well described by a one-zone SSC model. 


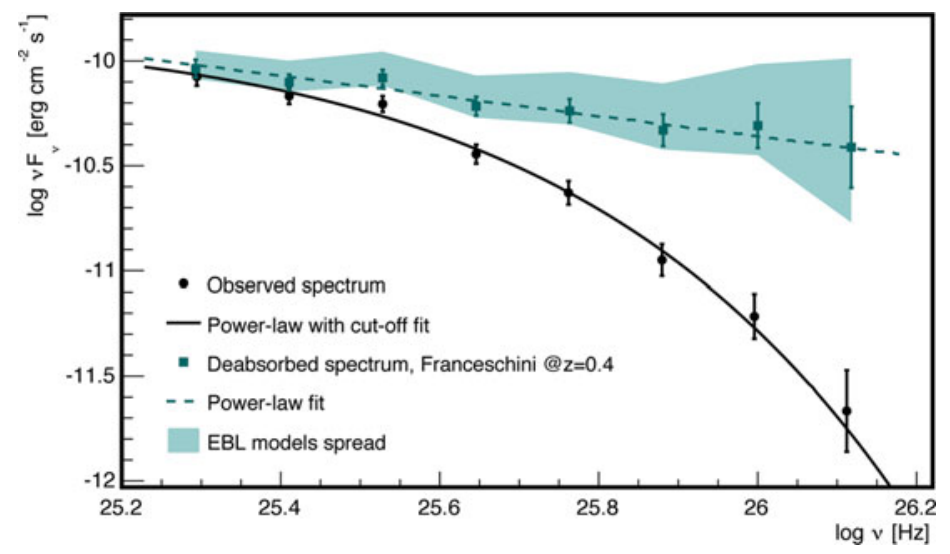

Figure 3. SED of PG $1553+113$ as measured by MAGIC during the flare state of 2012 April. The observed SED is shown as black circles, and the black solid line represents the best fit to a power-law with an exponential cut-off. The absorption-corrected spectrum assuming $\mathrm{z}=0.4$ and using the EBL model by Franceschini et al. (2008) is shown by the green squares; the dashed green line is the best-fitting power-law. The green shaded area account for the uncertainties derived by the use of different EBL models (Domínguez et al. 2011; Kneiske \& Dole 2010; Franceschini et al. 2008; Gilmore et al. 2012).

\section{Summary}

The MAGIC telescopes detected recently in 2014 July the first gravitational lensed blazar S3 0218+357 in the VHE band. Also two new BL Lac objects has been detected during 2014.

Fast intra-night variability have been found in IC 310. Due to its low Doppler factor with respect to the typical blazars, it points to emitting regions which are smaller than the event horizon being therefore difficult to be explained by the current models.

The long term MWL study of the BL Lac PKS 1424+240 shows correlation between the radio and optical flux and points to a common origin. The MWL observations also challenge the standard one-zone SSC model, and a two-zone model is found to describe the SED.

Spectral curvature was found in the observed VHE spectrum from the BL Lac PG $1553+113$. For the first time spectral curvature compatible with the EBL imprints was found in an individual target.

\section{Acknowledgements}

We would like to thank the Instituto de Astrofísica de Canarias for the excellent working conditions at the Observatorio del Roque de los Muchachos in La Palma. The support of the German BMBF and MPG, the Italian INFN, the Swiss National Fund SNF, and the ERDF funds under the Spanish MINECO is gratefully acknowledged. This work was also supported by the CPAN CSD2007-00042 and MultiDark CSD2009-00064 projects of the Spanish Consolider-Ingenio 2010 programme, by grant 127740 of the Academy of Finland, by the Croatian Science Foundation (HrZZ) Project 09/176, by the DFG Collaborative Research Centers SFB823/C4 and SFB876/C3, and by the Polish MNiSzW grant 745/N-HESS-MAGIC/2010/0. 


\section{References}

Ackermann, M. et al. 2013, ApJS, 209, 34

Aharonian, F. et al., 2007, ApJ, 664, L71

Albert, J. et al., 2007, ApJ, 669, 862

Aleksic, J. et al. 2010, ApJ 723, L20

Aleksić, J. et al. (MAGIC Collaboration), 2012, APh, 35, 435

Aleksić, J. et al. 2014a, arXiv:1409.5594

Aleksić, J. et al. 2014b, A\&GA, 563, 91

Aleksić, J. et al. 2014c, Science, 346, 1080

Aleksić, J. et al. 2014d, A\&A, 567, 15

Aleksić, J. et al. 2014e, arXiv:1408.1975

Buson, S., Cheung, C. C. \& LAT collaboration, 2014, ATel \#6316

Cheung, C. C. et al. 2014, ApJ, 782, L14

Danforth, C. W. et al. 2010, ApJ 720976

Domínguez, A. et al. 2011, MNRAS, 410, 2556

Franceschini, A., Rodighiero, G., \& Vaccari, M. 2008, A\&A, 487, 837

Furniss, A. et al. 2013, ApJL, 768, L31

Gilmore, R. C. et al. 2009, MNRAS, 399, 1694-1708

Hiroi, K. et al. 2013, ApJS, 207, 36

Kadler, M. et al. 2012, A\&BA, 538, L1

Kneiske, T. M. \& Dole, H., 2010, A\&A, 515, A19

Mirzoyan, R. et al. 2014a, ATel \#5768

Mirzoyan, R. et al. 2014b, ATel \#6062

Mirzoyan, R. et al. 2014c, ATel \#6349

Neronov, A. et al. 2010, A\&A 519, L6

Nolan, P. L. et al., 2012, ApJS, 199, 31

Ryle, M. \& Windram, M. D. 1968, MNRAS 138, 1 